\title{
Preliminary Detection of COVID-19 Using Deep Learning and Machine Learning Techniques on Radiological Data
}

\author{
Koti Neha \\ Assistant Professor, Computer Science and Engineering, \\ GITAM (deemed to be University), Hyderabad,502329, India. \\ nehak9104@gmail.com \\ Kundoju Param Joshi \\ Assistant Professor, Computer Science and Engineering, \\ GITAM (deemed to be University), Hyderabad,502329, India. \\ param.joshi1111@gmail.com \\ Nitturu Asha Jyothi \\ Assistant Professor, Computer Science and Engineering, \\ GITAM (deemed to be University), Hyderabad,502329, India. \\ nitturuashajyothi@gmail.com \\ Joshi Vinay Kumar \\ Assistant Professor, Computer Science and Engineering, \\ GITAM (deemed to be University), Hyderabad,502329, India. \\ joshivinay619@gmail.com
}

\begin{abstract}
Covid-19 is a dangerous pandemic in the year 2020." Covid-19 positive" is the most negative word heard this year, which caused terror worldwide. As it is a contagious pandemic, early detection of this pandemic will minimize its threat. The primary issue is its detection. To detect Covid-19 through a blood test, a person must wait for an extended period to get the results. Using our model, one can primarily detect Covid-19 immediately using Deep Learning algorithm CNN and Machine Learning algorithm Logistic Regression. Input to these techniques is radiological data like CT-Scan and X-ray images. Covid19 positive cases will be easily detected faster with the help of this model.
\end{abstract}

Keywords: Covid-19; Viral infection; Deep Learning; Conventional Neural Networks(CNN); Machine Learning; Logistic Regression; CT-Scan Images; X-ray Images.

\section{INTRODUCTION}

COVID-19 is one of the Corona family viruses, which is very dangerous and declared a global pandemic in 2020 by the World Health Organization (WHO Corona Virus Disease)[WHO(2020)]. It was first discovered in Wuhan, China, in December 2019. The spread[Arabella(2020)] of this disease can occur directly from infected humans or indirectly through contaminated objects or surfaces. Fever, tiredness, dry cough, chest pain, shortness of breath are some of the symptoms of this disease[Ahmet(2020)]. In most of the cases, symptoms[Muhammad Adan Shereen(2020)] are found out after fourteen days of infection. Covid-19 can infect lower and upper respiratory tracts at a higher rate when compared to other viruses[Jie cui(2019)]. It led to a community spread so fast that there are currently 68,567,527 [WorldOmeter(2020)] affected persons throughout the world. There is no vaccine currently which can cure COVID 19. Maintaining social distance, using masks and sanitizers are the only precautionary steps [Stephanie J. Dancer(2020)] people are following to avoid this disease to date.

Early detection of this disease can stop community spread. Due to this sudden pandemic, various research centers try to determine different diagnostic techniques and vaccines for curing it. Apart from the medical field, this research now expanded to other fields like Machine learning, Data Science, Deep Learning[Geert Litjens(2017)][Song Ying(2020)] and Artificial Intelligence[Arun Sharma(2020)] for faster-diagnosing methods. Many tests like RT-PCR[Chloe Kent(2020)]Tracheal aspirate, sputum test, Blood test to detect COVID 19[Shervin Minaee(2020)].need a few days to get results. These tests need samples of nasal secretions, mucus from the lungs, blood samples. Figure 1 below gives the microscopic image of COVID 19 (National Institutes of health, 2020). 


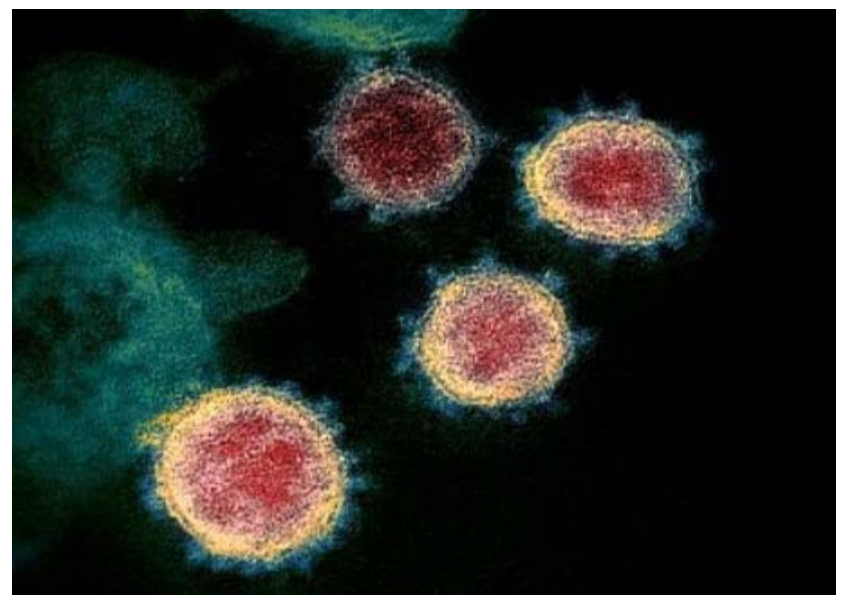

Fig.1. Microscope image of COVID 19

Many researchers have taken only one type of dataset from open literature, either CT-scan images or X-ray images [Mufti Mahmud(2020)][Maribel Torres-Velazquez(2020)], and given this dataset to only one algorithm.

The novelty of this paper is:

1. Our proposed model, takes two different input datasets: CT-scan Images and X-ray images.

2. CNN and Logistic Regression techniques are used to detect COVID 19 positive or negative for given input datasets.

3. Our model is cost-efficient and consumes less time to detect when compared to other types of blood tests.

\section{Related Work}

Many approaches have been proposed to detect covid-19 using Deep learning and Machine learning algorithms refer to Table 1. However, most of these models have used only one of the deep learning algorithms and only one type of dataset.

[Y. Pathak (2020)] proposed an approach named Deep Transfer Learning, to detect Covid-19 positive or negative. 852 CT-Scan images are taken as dataset. Initially this dataset is given to ResNet50 to extract desired features and next to train the dataset transfer learning is used. This model achieved $96.22 \%$ accuracy for training dataset and $93.01 \%$ accuracy for testing dataset.

[Harsh Panwar(2020)] proposed an approach named nCOVnet, to which 337 X-ray images are given as an input data set. Input images are of size 224 X 224 pixels. This model contains a total of 24 layers. VGG model is used to extract features. In the Pooling layer, Average pooling is taken. Adam Optimization technique is used for optimization. This model achieved training accuracy in the range of $93 \%-97 \%$. This model achieved $98.68 \%$ confidence for covid-19 negative patients and achieved $97.62 \%$ confidence for covid-19 positive patients.

[Moutaz Alazab (2020)] proposed a model to detect Covid-19 and predict the number of covid-19 cases in the next 7days. VGG16, a type of CNN, is used to detect Covid-19. $1000 \mathrm{X}$-ray images are taken as an input to the model. $80 \%$ of the dataset is used for training. The rest, $20 \%$, is used for testing. They achieved an F-measure range of $95 \%$ accuracy on the augmented dataset.

[Xuehai He (2020)] proposed a model named the Self-Trans Approach to detect covid-19. This model is a combination of transfer learning and self-supervised learning. 349 Covid-19 positive CT-Scan images and 397 Covid-19 negative CT-Scan images are taken as the dataset. In this model, Labels are not given manually. The system detects features by itself in an image and tries to categorize them. This model achieved $86 \%$ overall accuracy and also achieved F1 with $85 \%$ and AUC with $94 \%$ in diagnosing Covid-19 patients.

[Chuansheng Zheng (2020)] proposed the DeCoVNet model, which detects Covid-19 in CT-Scan images. A total of 499 CT-Scan images were taken as the initial dataset. After data pre-processing and Augmentation, the output 3D lung mask of images are given as input to their model, which detects the probability of being Covid-19 positive or negative. They achieved an accuracy of $95.9 \%$ ROC AUC, 97.6\% PR AUC.

[Govardhan Jain (2020)] proposed a model that first detects whether it is bacterial or viral pneumonia, and next, if it is viral, again detects whether it is caused by a covid-19 virus or by other viruses. Total $1832 \mathrm{x}$-ray images are taken as the initial dataset. This model is divided into four steps. First, the data augmentation step, where x-ray images are resized into $640 * 640$ size. The next step is data pre-processing, in which images are rotated, and the Gaussian blur method is applied to images. The third step is Stage-I, where the ResNet50 method is used to find whether it is a viral disease or bacterial disease. If it is viral pneumonia in stage-II, which is the last step of this model, uses ResNet101 network to detect if it is caused by Covid-19 or not. This model achieved $97.77 \%$ accuracy. 
[Mohamed Abd Elaziz (2020)] proposed a model to detect Covid-19. . Two datasets were taken. The first dataset contains 216 Covid-19 positive X-ray images and 1675 negative X-ray images. The second dataset contains 219 Covid-19 positive X-ray images and 1341 negative X-ray images. These input images are given to the Fractional Multichannel Exponent Moments (FrMEMs) model to extract 961 features from each x-ray image. Then KNN classifier is used for training the dataset and then detects whether Covid-19 positive or negative image based on the extracted features. This model achieved $96.09 \%$ accuracy for the first dataset and $98.09 \%$ accuracy for the second dataset.

[LinLi (2020)] proposed a model named COVNet to detect Covid-19 positive or negative CT-Scan images. Total 4352 chest CT-Scan images are taken as a dataset. First images are pre-processed and converted into the same size as $512 * 512$ pixels. Then these resized images are given to the COVNet model, which detects whether a given CT-Scan image belongs to the Covid-19 positive or negative group.

\section{Proposed Algorithm}

\subsection{Datasets}

Many datasets that contain Covid-19 medical images are available now. Images are collected from Kaggle, an open-source database for our model. Radiological data are easy to obtain and are cheap when compared to other Covid-19 detection tests. For our model, two different types of image datasets are taken: Chest CT-Scan images and X-ray images.

\subsubsection{Covid-19 CT-Scan Images Dataset :}

CT-Scan Images[Abu Zahid Bin Aziz (2020)] of a total of 1000 are given as input to our model. Among these, 500 are Covid-19 positive images, and 500 are Covid-19 negative images.

\subsubsection{Covid-19 X-ray Images Dataset :}

We have taken 1000 X-ray images[Nabeel Sajid(2020)] as another type of input dataset[Larxel(2020)] Among them, 500 are Covid-19 positive, and the rest 500 are Covid-19 negative images. $80 \%$ from both the datasets are used for training the model, and $20 \%$ are used for testing. Some of the sample images of both datasets are shown in Figure 2 below.

$\mathbf{W}$

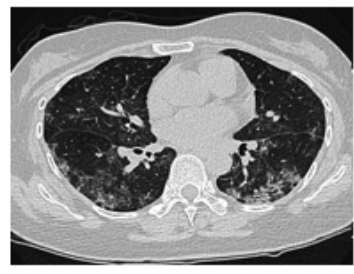

$\mathbf{X}$
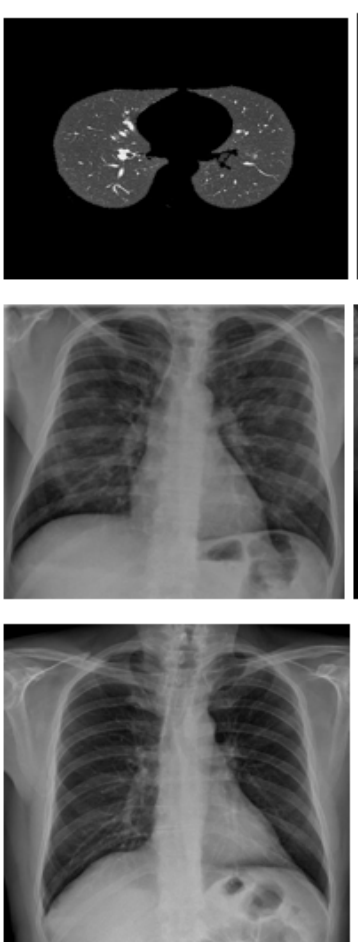
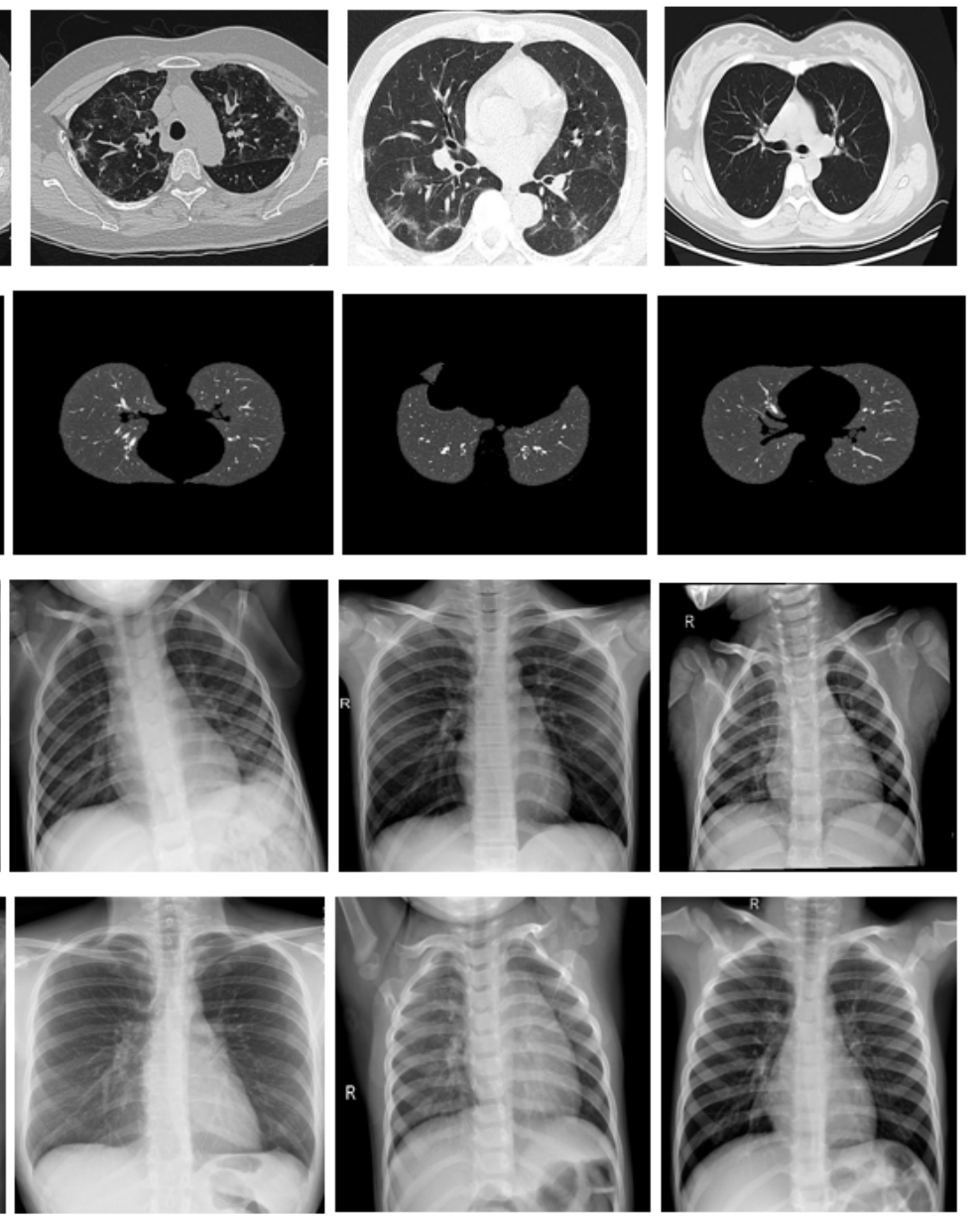

Fig.2.Sample datasets where,W: Sample Training set CT-Scan images, X: Sample Testing 


\subsection{Proposed Model}

The proposed model is divided into three modules, namely Image pre-processing, CNN module, Logistic Regression module, refer to Figure 5 below.

\subsubsection{Image Pre-processing:}

Input datasets contain CT-Scan and X-ray images of different sizes. We resize all images into the same pixel size of 512 X 512. Next, these resized images are converted into Grayscale Images refer Figure 3 below. We divide 1000 CT-Scan images into two parts: 800 images as the training set and 200 images as the test set. Similarly, 1000 X-ray images are divided into two parts: $800 \mathrm{X}$-ray images for the training set and $200 \mathrm{X}$-ray images for the test set. Furthermore, these images are categorized into two labels, namely, positive and negative.

\subsubsection{CNN Module:}

From the Image pre-processing step, we get Grayscale CT-Scan images and X-ray images of size 512 X 512 . We have an input layer, 15 layers (a combination of Convolutional, ReLu, and Max-pool layers), and an output layer in CNN. First, we pass Training datasets of CT-Scan images and X-ray images with already predefined labels. Now pass Testing datasets to Convolutional+ ReLu layer. Then we pass this output image from the first layer to the Max Pooling layer. Like this, we repeat these layers five times to get an 8 X 8 image as output. We give these output images from the last 15 th layer to the Fully connected layer, which categorizes the given image to either Covid-Positive or Covid-Negative label and finally passed to the output layer to display it(refer to steps 1 to 6 in the below algorithm. Detailed explanation of CNN is described in below.
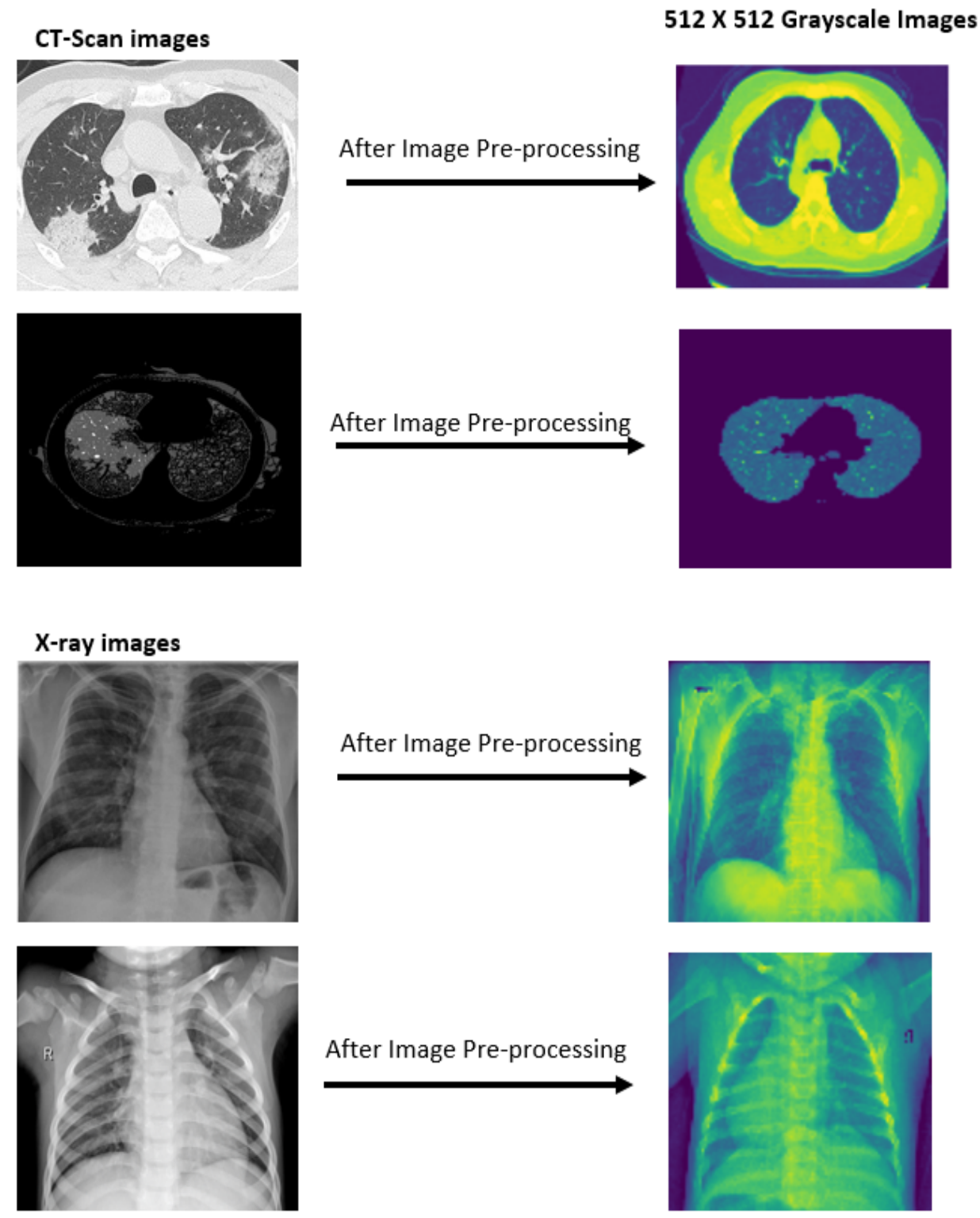

Fig.2.Sample Images in Image Pre-processing Module 
Convolutional Neural Network (CNN) or ConvNet [Rikiya Yamashita(2020)][Ahmed S.Shamsaldin(2019)] is one of the most efficient and successful deep learning models used in medical research. From the Visual cortex (which processes visual information) of an animal's brain, we got CNN's motivation. It is used to analyze patterns in images. Input to $\mathrm{CNN}$ model are images passed through some hidden layers called Convolutional layers, and finally, we get output that classifies or labels the given input image based on the training data given to CNN. The number of parameters required for ConvNet is significantly less than a Fully connected network, and thus CNN does not have an Overfitting problem. ConvNet has four essential layers: The Convolution layer, the ReLU layer, the pooling layer, and the fully connected layer. Each layer uses filters to process the given input image. We have different filters that detect small details like edges, corners, circles, and squares in an image or detect special complex features like eyes, nose, dogs, trees, and cats. We get more sophisticated filters as the number of layers increase. Accuracy will be more if we include more layers in our model. Computers understand images using numbers at each pixel, containing either RGB pixel values (3 channels) or Black and white pixel values (2 channels). The size of any image will be represented as $\mathrm{X} * \mathrm{Y} * \mathrm{Z}$, where $\mathrm{X}$ indicates the number of rows, $\mathrm{Y}$ indicates the number of columns, and $\mathrm{Z}$ indicates the number of channels.

CNN [Shaik Basheera (2020)][Michael Gadermayr (2020)] works like this: an image of size $\mathrm{X} * \mathrm{Y} * \mathrm{Z}$ is given as an input to CNN. This image is passed to the first layer, called the Convolution layer. We need to specify the number of filters to be used in each layer. We pass the input image to each filter. A filter is represented as a small matrix that contains $\mathrm{n} * \mathrm{n}$ rows and columns, and the elements in the matrix contain number values of features. For example, for black-colored pixels, we use 1 (one number); for white-colored pixels, we use 0 (zero number). We take a small part or patch of the input image and perform a dot product or multiply corresponding pixel values of this small part of the image with the corresponding filter or feature matrix. Then we add all these values of the resultant and divide it by the total number of pixels in the feature matrix. Like this, we take a feature or filter and apply it across every possible patch across the whole image and see how the feature matches that area refer to Figure 4 below. We get a transformed image as an output of this layer with new pixel values. We give this new transformed image to the next layer that is ReLU layer.
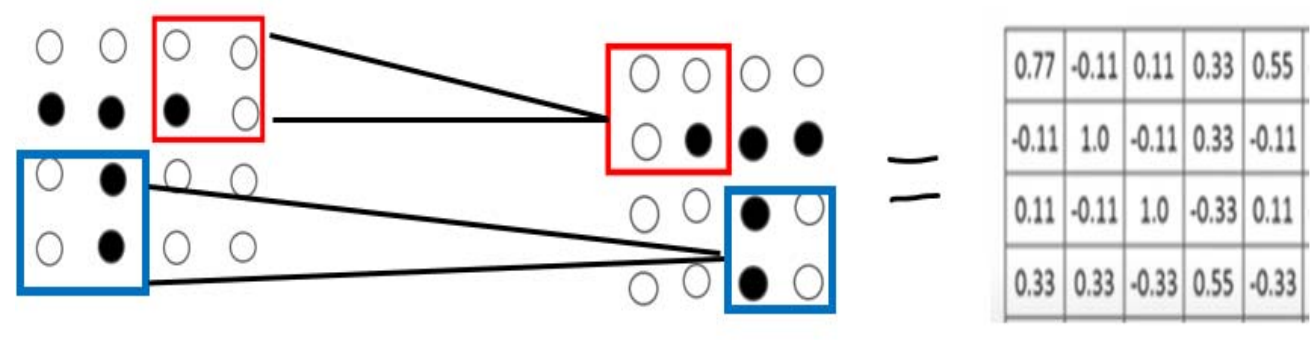

Fig.3.Feature Extraction in Convolutional Layer

ReLU layer is also known as the activation layer, where we remove negative values in the resultant matrix we got from the convolutional layer and replace them with zeros. This is done to avoid values from summing up to zero. The Rectified Linear Unit (ReLU) function activates a node only when the function value is above some threshold value. Otherwise, the output will be zero refer equation (1).

$$
f(a)= \begin{cases}0 \text { if } a<0 \\ \text { a if } a>=0\end{cases}
$$

The resultant matrix from the ReLU layer is given to the Pooling layer, which resizes and shrinks the input image. We have a window size, usually of 2 or 3, moved throughout the resultant image we got from the ReLU layer. We reduce the input image's size by repeating the above procedure throughout the input image. Max Pooling and Average Pooling are two types of Pooling techniques. Max Pooling takes the maximum value within the window, whereas Average pooling takes the average of all values present within the window. Max pooling is better than Average Pooling. We can repeat these three layers convolutional layer, ReLU layer, and Pooling layer in various iterations to get a small size image. This reduced image is given as an input to a fully connected layer. The actual classification happens in this layer. We write all values of all filters of the shrunk image into a 1D array or single list. We compare these list values with already trained image list values then classify accordingly.

\subsubsection{Logistic Regression Module}

Training datasets are passed to Logistic function or Transfer function to get maximum cost. If this cost is greater than the threshold value, it is mapped to 1 value, which is assigned to the Covid-positive label. If the cost is less than the threshold value, it is mapped to 0 value, which is assigned to the Covid-Negative label(refer to steps 7 to 12 in the below algorithm). Detailed explanation of Logistic regression is described below. 
Logistic Regression is one of the techniques of Regression Analysis, which is mainly used for binary classification[Pranav Rao(2017)]. It is also used in statistics and multiclass classification. Logistic Regression produces results[Lin $\mathrm{Li}(2020)]$ in a binary format, which is used to predict the outcome or probability of a categorical dependent variable. So the outcome is discrete such as 0 or 1, True or False, Yes or No. Logistic Regression is of three types: Binary ( where the dependent variable can have only two possibilities like it will rain or not, Spam or not examples), Multinomial (where the dependent variable can have three or more possibilities like identifying birds, cats, dogs images, type a or b or c kind of examples), Ordinal ( where the dependent variable is classified into one of the ordered categories like Exam score can be categorized into below average, average, excellent)[R. P. Jaia Priyankka (2020)].

The logistic function uses the Sigmoid function to predict the probability of the given data. The sigmoid function is also known as the logistic function, which is defined as below equation (2):

$$
\operatorname{sigmoid}(z)=\frac{1}{(1+e-z)}
$$

where "e" is Euler's number equivalent to 2.71828, " $\mathrm{z}$ " is straight-line equation. When a graph is plotted, this function looks like an "S" shaped curve refer Figure 5 below.

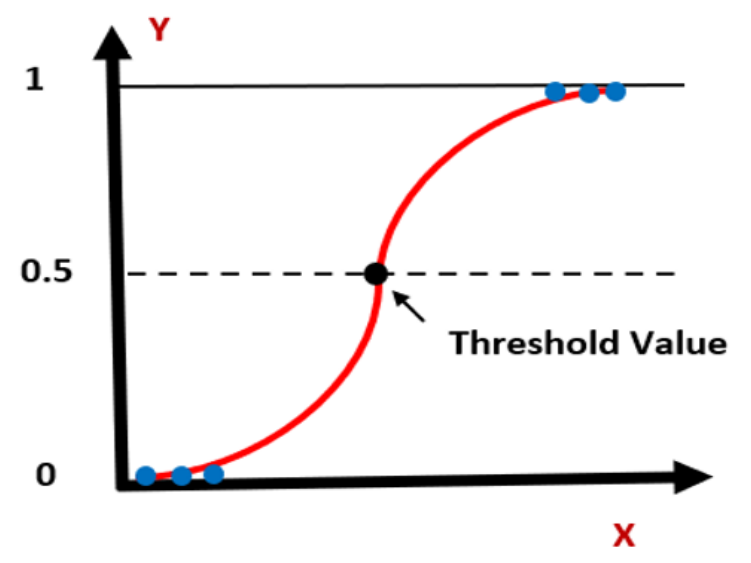

Fig.4.Logistic Function

We give inputs to this logistic function, and it will convert them within the range of 0 and 1 . A threshold value will be fixed with this range of 0 and 1 . And based on this threshold value, the new input will be classified into one of the labels which are already specified in the problem.

\subsubsection{Proposed Algorithm}

\section{Parameters Used:}

$\Delta 1 \rightarrow$ Training set of CT-Scan Grayscale images , $\Delta 2 \rightarrow$ Testing set of CT-Scan Grayscale images

$\lambda 1 \rightarrow$ Training set of X-ray Grayscale images, $\lambda 2 \rightarrow$ Testing set of X-ray Grayscale images

Convolutional Neural Network $\rightarrow \mathrm{C}_{\mathrm{N}}$, Logistic Regression $\rightarrow \mathrm{L}_{\mathrm{G}}$

$\mathrm{i} \rightarrow$ iteration variable, $\alpha \rightarrow$ Threshold Value

Begin:

Step 1: Give $\Delta 1, \lambda 1$ to $\mathrm{C}_{\mathrm{N}}$

Step 2: for $i=1$ to 5 repeat

Pass each image in $\Delta 1, \lambda 1$ to Convolutional + ReLu layer

Pass the Outputs from above layer as input to Max Pooling layer

Step 3: Output of Max pooling layer is given as input to Fully connected layer. (we completed Training the Model in $\mathrm{C}_{\mathrm{N}}$ )

Step 4: Now Pass $\Delta 2, \lambda 2$ to $\mathrm{C}_{\mathrm{N}}$ to validate the model.

Step 6: Each image in the Testing dataset is categorized as either Covid-positive or Covid-negative label.

Similarly,

Step 7: Give $\Delta 1, \lambda 1$ to $\mathrm{L}_{\mathrm{G}}$

Step 8: Calculate Transfer Function: $\beta=\mathrm{n} 0+\mathrm{n} 1 \mathrm{X}$ 
Step 9: Apply Transfer function to find Max cost:

$$
\begin{aligned}
& h(X)=\text { Transfer(B) } \\
& h(X)=1 /\left(1+e^{n 0+n 1 X}\right)
\end{aligned}
$$

Step 10: If output of $h(X)>\alpha$, label $=1$

Else:

$$
\text { label }=0 \text {. }
$$

(We Completed training the model in $\mathrm{L}_{\mathrm{G}}$ )

Step 11: Now pass $\Delta 2, \lambda 2$ to $\mathrm{L}_{\mathrm{G}}$ to validate the model.

Step 12: Given input images are categorized as:

If label $=0$, then it is Covid-Negative

Else if label $=1$, then it is Covid-Positive.

End

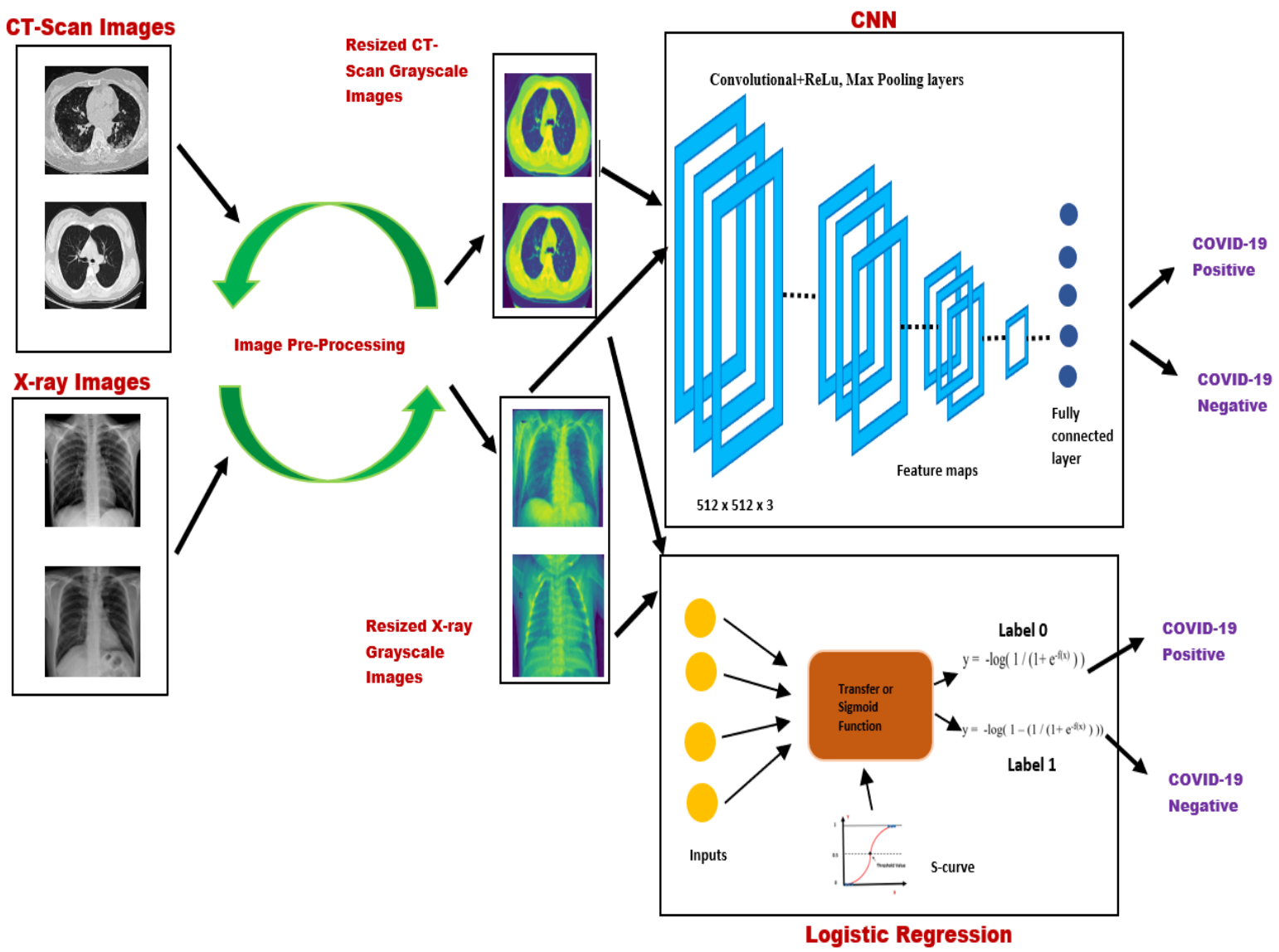

Fig.5.Architecture of Proposed Model

\subsection{Performance Metrics}

4. Experiment and Results

The performance of our proposed model is measured using metrics like Confusion matrix, Accuracy[Mohammed Sunasra(2017)], Precision, Recall, F1 Score refer Table 1 below.

4.1.1. Confusion Matrix: It contains four parameters that are used to predict the overall performance of any model. They are: True Positive(o): Total number of positive cases which are actually positive in nature, True Negative $\left(\boldsymbol{\theta}^{\prime}\right)$ : Total Number of negative cases which are actually negative in nature, False Positive $(\boldsymbol{\alpha})$ : Total number of positive cases which are actually negative in nature, False Negative $\left(\boldsymbol{\alpha}^{\prime}\right)$ : Total number of negative cases which are actually positive in nature.

4.1.2.Accuracy: It is defined as Summation of True positives and True Negatives divided by total number of samples in the dataset refer equation (3).

$$
\text { Accuracy }=\left(\Theta+\theta^{\prime}\right) / \text { Total No. of samples }
$$


4.1.3.Precision: It is defined as division of total true positives by sum of total true positives and total false positives. refer equation (4)

$$
\operatorname{Precision}(\mathrm{P})=\left(\Theta /\left(\theta^{+}+\alpha\right)\right)
$$

4.1.4.Recall: It is defined as division of total true positives by sum of total true positives and total false negatives. refer equation (5)

$$
\text { Recall }(\mathrm{R})=\left(\Theta /\left(\theta^{+}+\alpha^{\prime}\right)\right)
$$

4.1.5.F1 Score: It is calculated using precision and recall values as below: refer equation (6)

$$
\mathrm{F} 1=2 *(1 /(1 / \mathrm{P})+(1 / \mathrm{R}))
$$

Table 1: Summary of different Performance Metrics

\begin{tabular}{|lcccccccc|}
\hline \multicolumn{1}{|c}{ Type } & TP & TN & FP & FN & Precision & Recall & F1-Score & Accuracy \\
\hline CNN(CT-Scan dataset) & 494 & 497 & 3 & 6 & 0.993963 & 0.988000 & 0.495486 & 0.991000 \\
\hline CNN(X-ray dataset) & 483 & 490 & 10 & 17 & 0.979716 & 0.966000 & 0.486405 & 0.973000 \\
\hline Logistic Regression (CT-Scan dataset) & 490 & 494 & 6 & 10 & 0.987903 & 0.980000 & 0.491967 & 0.984000 \\
\hline Logistic Regression(X-ray dataset) & 480 & 483 & 17 & 20 & 0.965794 & 0.960000 & 0.481444 & 0.963000 \\
\hline
\end{tabular}

\subsection{Comparative Analysis:}

Our proposed model performance is evaluated based on above performance metrics. For the Convolutional Neural Network module on the CT-Scan images dataset, we achieved $99.10 \%$ accuracy, and for the X-ray images dataset, we achieved 97.36\% accuracy. For the Logistic Regression module on the CT-Scan images dataset, we achieved $98.46 \%$ accuracy, and for the X-ray images dataset, we achieved $96.35 \%$ accuracy. Our proposed model achieved better performance than few other models mentioned in the Related work in the above section.

Table 2: Summary of different COVID-19 Detection Methods

\begin{tabular}{|lccc|}
\hline \multicolumn{1}{|c}{ Model } & No. of Images & Image Type & Accuracy \\
\hline nCOVnet & $\mathbf{3 3 7}$ & X-ray & $\mathbf{9 8 . 6 8 \%}$ \\
\hline VCC16 & 1000 & X-ray & $95.00 \%$ \\
\hline Self-Trans Approach & 746 & CT - Scan & $86.00 \%$ \\
\hline DeCOVnet & 499 & CT- Scan & $97.60 \%$ \\
\hline ResNet50, ResNet101 & 1832 & X-ray & $97.77 \%$ \\
\hline FrEMEMs, KNN classifier & 3016 & X-ray & $98.09 \%$ \\
\hline COVNet & 4352 & CT-Scan & $96.00 \%$ \\
\hline Deep Transfer Learning & $\mathbf{8 5 2}$ & CT-Scan & $\mathbf{9 6 . 2 2 \%}$ \\
\hline Proposed Model(CNN Module) & $\mathbf{5 0 0}$ & CT-Scan & $\mathbf{9 9 . 1 0 \%}$ \\
\hline Proposed Model(CNN Module) & $\mathbf{5 0 0}$ & X-ray & $\mathbf{9 7 . 3 0 \%}$ \\
\hline Proposed Model(Logistic Module) & $\mathbf{5 0 0}$ & CT-Scan & $\mathbf{9 8 . 4 0 \%}$ \\
\hline Proposed Model(Logistic Regression Module) & $\mathbf{5 0 0}$ & X-ray & $\mathbf{9 6 . 3 0 \%}$ \\
\hline
\end{tabular}




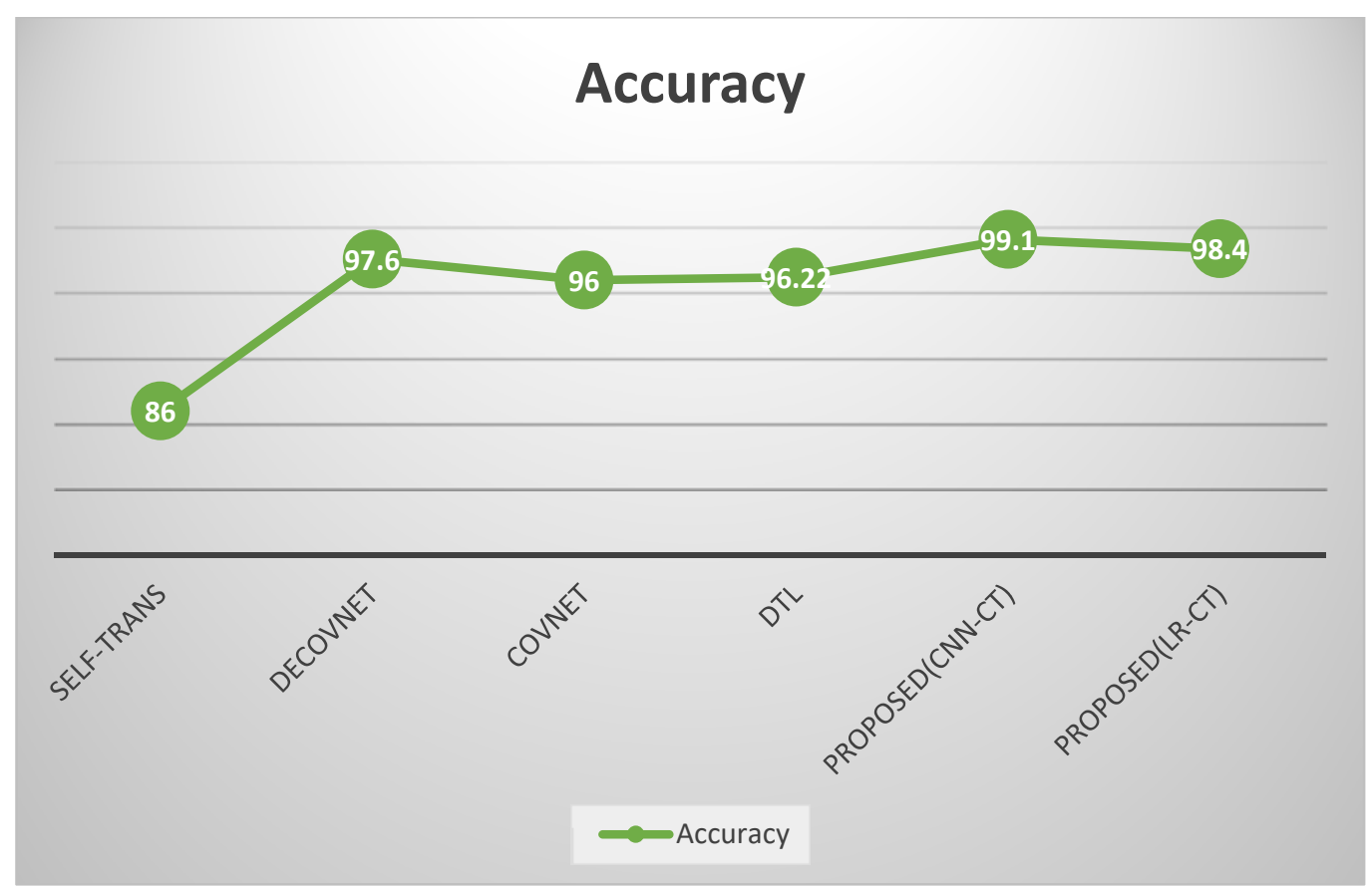

Fig.6. Comparison Graph of Various Models Using CT-Scan Datasets

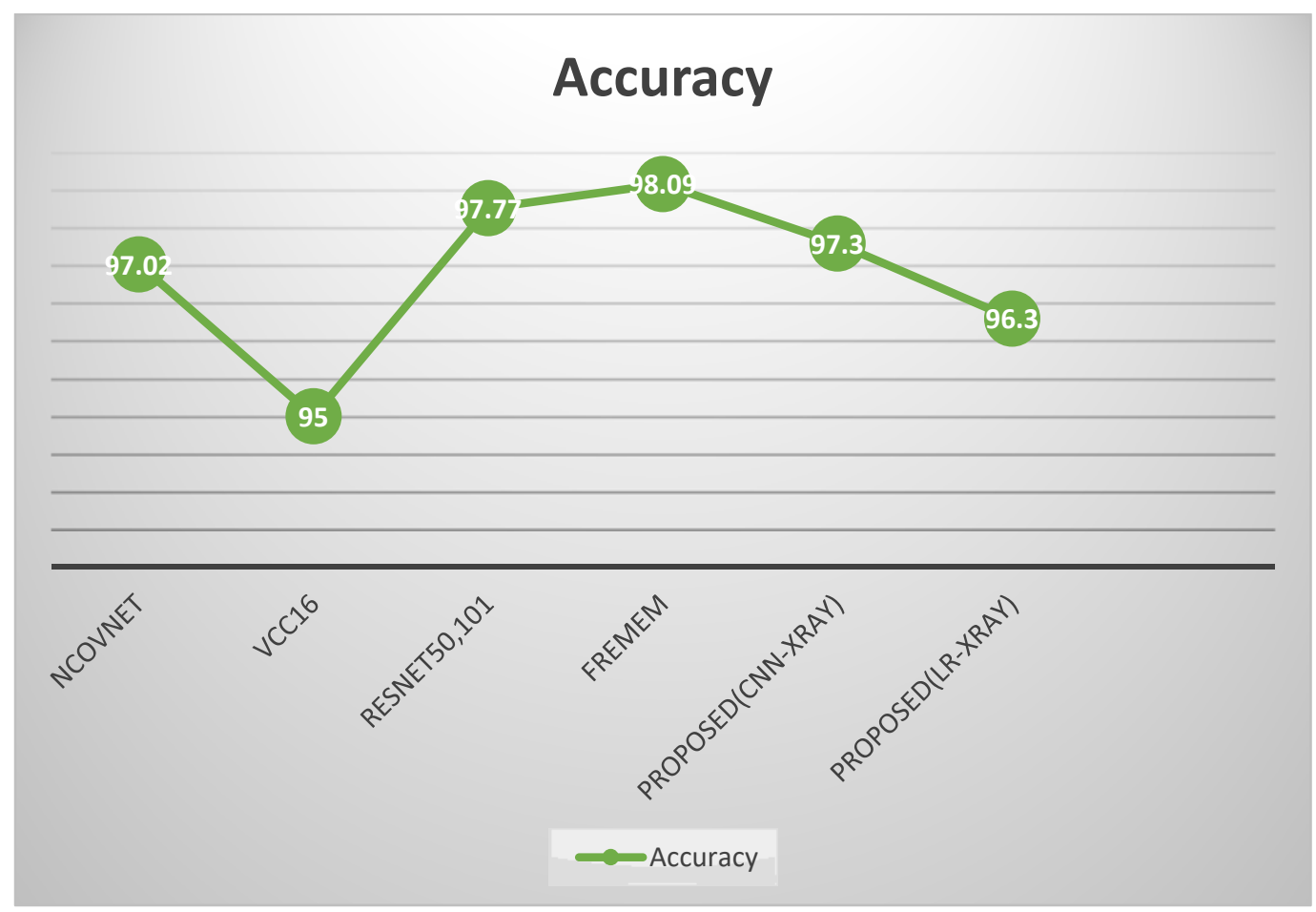

Fig.7. Comparison Graph of Various Models Using X-Ray Datasets

Among both models, CNN module achieved better accuracy than the Logistic Regression module. Furthermore, within two different datasets taken, CT-Scan images datasets achieved higher accuracy than X-ray images datasets refer Table 2 and Figures 6,7 above.

\section{CONCLUSTION}

Prediction of Covid-19 as early as possible is necessary to stop its spread. Using deep learning and machine learning techniques like CNN and Logistic Regression, we can easily predict Covid-19 with radiological data like CT-Scan and X-ray images faster when compared to blood tests. Many models have been proposed; among them, our proposed model also, could able to detect covid-19 positive or negative, with reasonable and better accuracy when compared to other models for both CT-Scan and X-ray datasets. In Future work, we can find out the severity of the disease if detected as COVID-19 Positive. 


\section{References}

[1] T. Pathak, P. K. Shukla, A. Tiwari, S. Stalin, S. Singh (2020).Deep Transfer Learning Based Classification Model for COVID-19 Disease', Innovation and Research in Bio-Medical Engineering.

[2] Harsh Panwar, P.K. Gupta, Mohammad Khubeb Siddiqui, Ruben Morales-Menendez, Vaishnavi Singh (2020) .Application of deep learning for fast detection of COVID-19 in X-Rays using nCOVnet, Chaos Solitons and Fractals, Volume 138.

[3] Moutaz Alazab, Albara Awajan, Abdelwadood Mesleh, Ajith Abraham, Vansh Jatana, Salah Alhyari (2020) COVID-19 Prediction and Detection Using Deep Learning,International Journal of Computer Information Systems and Industrial Management Applications, Vol.12.

[4] Geert Litjens, Thijs Kooi, Babak Ehteshami Bejnordi, Arnaud Arindra Adiyoso Setio, Francesco Ciompi, Mosen Ghafoorian, Jeroen A.W.M van der Laak, Bram van Ginneken, Clara I. Sanchez (2017).A Survey on deep learning in medical image analysis, Medical Image Analysis. Volume No:42.

[5] Xuehai He, Xingyi Yang, Shanghang Zhang, Jinyu Zhao, Yichen Zhang, Eric Xing, Fellow,IEEE, Pengtao Xie (2020).Sample-Efficient Deep-Learning for COVID-19 Diagnosis Based on CT Scans. IEEE TRANSACTIONS ON MEDICAL IMAGING.

[6] Chuansheng Zheng, Xianbo Deng, Qiang Fu, Qiang Zhou, Jiapei Feng, Hui Ma, Wenyu Liu, Xinggang Wang (2020). Deep-LearningBased Detection for COVID-19 from Chest CT using Weak Label.

[7] Govardhan Jain, Deepti Mittal, Daksh Thakur, Madhup K. Mittal (2020).A Deep-Learning Approach to Detect Covid-19 Coronavirus with X-Ray images, ScienceDirect,Volume 40.

[8] Mohamed Abd Elaziz, Khalid M. HosnyID, Ahmad Salah, Mohamed M. Darwish, Songfeng Lu Ahmed T. Sahlol (2020). New machine learning method for image-based diagnosis of COVID-19.

[9] Lin Li, Lixin Qin, Zeguo Xu, Xin Wang, etc (2020). Using Artificial Intelligence to Detect COVID-19 and Community-acquried Pneumonia Based on Pulmonary CT: Evaluation of the Diagnostic Accuracy, Thoracic Imaging.

[10] Shaik Basheera, M.Satys Sai Ram (2020). A Novel CNN Based Alzheimer's disease classification using hybrid enhanced ICA Segmented gray matter of MRI. Computerized Medical Imaging and Graphics, Volume 81.

[11] Rikiya Yamashita, Mizuho Nishio, Richard Kinh Gian Do, Kaori Togashi (2020). Convolutional neural networks: an overview and application in radiology Insights Into Imaging.

[12] Shervin Minaee, Rahele Kafieh, Milan Sonka, Shakib Yazdani, Ghazaleh Jamalipour Soufi (2020). Deep-COVID: Predicting COVID19 from chest X-ray images using deep transfer learning.

[13] National Institute of Health(March,2020) [Online], https://www.nih.gov/news-events/nih-research-matters/novel-coronavirus-structurereveals-targets-vaccines-treatments

[14] Abu zahid Bin Aziz (2020), CT Scans for COVID-19 Classification [Online], https://www.kaggle.com/azaemon/preprocessed-ct-scansfor-covid19

[15] Nabeel Sajid (2020), COVID-19 Patients Lungs X Ray Images 10000 [Online], https://www.kaggle.com/nabeelsajid917/covid-19-xray-10000-images.

[16] Larxel(2020),“COVID-19 X rays, [Online], https://www.kaggle.com/andrewmvd/convid19-X-rays?select=X+rays

[17] World Health Organization, WHO Coronavirus Disease,[Online], https://covid19.who.int/?gclid=CjwKCAiAwrf\%20\%20BRA9EiwAUWwKXpCzpxW61XJ0hJpM6QFM9MdAAy3w-yr8Dxh4xxz3sPAazZR9ok-exoC1KkQAvD_BwE

[18] WorldOmeter, COVID-19CORONAVIRUS PANDEMIC, [Online] https://www.worldometers.info/coronavirus/

[19] .Song Ying, Shuangjia Zheng, Liang Li ,etc (2020). Deep learning enables accurate diagnosis of novel coronavirus (covid-19) with ct images., medRxiv,

[20] Maribel Torres-Velázquez, Wei-Jie Chen, Xue Li, Alan B. McMillan (2020). Application and Construction of Deep Learning Networks in Medical Imaging.

[21] Ahmed S. Shamsaldin, Pola Fattah, Tarik A. Rashid, Nawzad K. Al-Salihi (2020). A Study of the Applications of Convolutional Neural Networks. UKH Journal of Science and Engineering, vol. 3, no. 2.

[22] Pranav Rao, Manikandan JÂ (2017). Design and Evaluation of Logistic Regression Model for Pattern Recognition Systems. IEEE Annual India Conference (INDICON).

[23] Mufti Mahmud, M Shamin Kiser, Amir Hussain (2020). Deep-Learning in Mining Biological Data.

[24] Mohammed Sunasra (2017). Performance Metrics for classification Problems in Machine Learning.

[25] Chole Kent (2020). Medical Device Network.

[26] Arun Sharma, Sheeba Rani, Dinesh Gupta (2020). Artifical Intelligence-Based Classification of ChesX-Ray Images into COVID-19 and Other Infectious Diseases. International Journal of Biomedical Imaging.

[27] Muhammad Adan Shereen, Suliman Khan, Abeer Kazmi, Nadia Bashir, Rabeea Siddique (2020). COVID-19 infection: Origin, transmission, and characteristics of human coronaviruses. Journal of Advanced Research 24, page no:91-98.

[28] Jie Cui, Fang Li, Zheng-Li Shi (2019). Origin and Evolution of Pathogenic Coronaviruses. Nature Reviews MicroBiology, Vol no :16, page no:181-192.

[29] Kartik Chopra, C. Srimathi, (2018). Logistic Regression and Convolutional Neural Networks Performance Analysis based on Size of Dataset, International Journal of Engineering Development and Research, Vol.no:6, Issue 1.

[30] R.P. Jaia Priyankka, S. Arivalagan, P. Sudhakar (2020). Deep Convolution Neural Network With Logistic Regression Based Image Retrieval And Classification Model For Recommendation System, INTERNATIONAL JOURNAL OF SCIENTIFIC \& TECHNOLOGY RESEARCH, VOLUME 9, ISSUE 01.

[31] Michael Gadermayr, Ann-Kathrin Dombrowski, Barbara Mara Klinkhammer, Peter Boor, Dorit Merhof (2019). CNN Cascades for Segmenting Sparse objects in gigaPixel Whole Slide images, Computerized Medical Imaging and Graphics, Vol.no:71, page no:40-48.

[32] Arabella Widders, Alex Broom, Jennifer Broom (2020). SARS-CoV-2: The Virual shedding Vs Infectivity dilemma. Infection, Disease $\&$ Health, Volume 25, issue 3, Page no: 210-215.

[33] Stephanie J. Dancer, Lanarkshire (2020). Covid-19 exposes the gaps in infection prevention and control, ScienceDirect. Volume 25, Page no: 223-226.

[34] Ahmet Qzyigit (2020). Understanding Covid-19 Transmission: The effect of temperature and health behaviour on transmission rates, ScienceDirect, Volume 25, page no:223-228. 